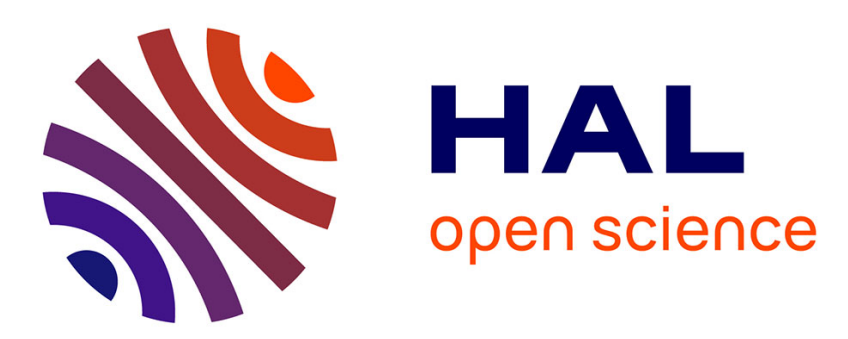

\title{
State Observation of a Specific Class of Unknown Nonlinear SISO Systems using Linear Kalman Filtering
}

Fawzia Amokrane, Emmanuel Piat, Joël Abadie, Adrien Drouot, Juan Antonio Escareno

\section{- To cite this version:}

Fawzia Amokrane, Emmanuel Piat, Joël Abadie, Adrien Drouot, Juan Antonio Escareno. State Observation of a Specific Class of Unknown Nonlinear SISO Systems using Linear Kalman Filtering. Conference on Decision and Control, Dec 2019, Nice, France. 10.1109/CDC40024.2019.9029614. hal-03053028

\section{HAL Id: hal-03053028 https://hal.science/hal-03053028}

Submitted on 10 Dec 2020

HAL is a multi-disciplinary open access archive for the deposit and dissemination of scientific research documents, whether they are published or not. The documents may come from teaching and research institutions in France or abroad, or from public or private research centers.
L'archive ouverte pluridisciplinaire HAL, est destinée au dépôt et à la diffusion de documents scientifiques de niveau recherche, publiés ou non, émanant des établissements d'enseignement et de recherche français ou étrangers, des laboratoires publics ou privés. 


\title{
State Observation of a Specific Class of Unknown Nonlinear SISO Systems using Linear Kalman Filtering
}

\author{
Fawzia Amokrane, Emmanuel Piat, Joël Abadie, \\ Adrien Drouot, and Juan Escareno
}

\begin{abstract}
Observing the state of totally unknown nonlinear systems is a problem that is addressed in the ADRC framework based on the use of Extended State Observers (ESO). A weak point of available ESO designs is that they do not take into account explicitly the statistical knowledge on the noise measurement when this one is available. This paper introduces a generic approach that makes possible to replace the ESO observer by a Linear Kalman filter, taking into account the variance of any Gaussian measurement noise. This approach can be applied on a specific class of unknown nonlinear SISO systems. Despite the fact that a linear Kalman filtering is a model-based estimation, the proposed approach makes possible the observation of nonlinear and time-varying systems when no information exists on their structure, time-varying parameters and potential disturbances. The process noise associated to this linear observation approach is also provided.
\end{abstract}

Index Terms - Observer for Nonlinear Systems, Extended State Observer, Kalman Filter, ADRC, Time-Varying Systems

\section{INTRODUCTION}

There exist various strategies for the obervation of nonlinear systems like the extended Kalman filter or one of its many extensions [11], Lie algebra-based observers [15], [6], Luenberger-like observers [28], [20], optimization-based observers [1], high-gain observers [13], etc. As the observers designed with these approaches duplicate the system's nonlinear dynamics, a basic requirement is that this dynamics is known to some extent. But in some cases the system cannot be modeled nor identified for a question of available time, cost, skills, etc. In this particular configuration, the system is considered as unknown but still requires observation and control schemes. Such considered unknown systems can be nonlinear and time-varying. In the field of control theory, the Active Disturbance Rejection Control (ADRC) framework [5], [4], [8] has been developed to overcome these problems without any training approaches. ADRC takes its deep roots in the Extended State Observer (ESO) [9] that is estimating an extended state that lumps unmodeled dynamics and external disturbances. Following the ESO parameterization steps described in [3], the ADRC technique has been used to solve various kinds of engineering problems [2], [27], [25]. Efficiency of ESO designs has been improved other

\footnotetext{
This work is supported by the EIPHI project, grant \# 17-EURE-0002. Fawzia Amokrane, Emmanuel Piat, Joël Abadie and Adrien Drouot are with the FEMTO-ST Institute, UMR CNRS 6174, UFC - ENSMM - UTBM, Besançon, France. emmanuel.piateens2m.fr, fawzia.amokrane@femto-st.fr, joel.abadied femto-st.fr, adrien.drouotefemto-st.fr

Juan Escareno is with the XLIM laboratory, UMR CNRS 7252, ENSIL - ENSCI, 16 rue Atlantis, Parc Ester Technopole, 87068 Limoges, France. juan.escareno-castro@unilim.fr
}

the years by increasing their order and that idea paved the way of higher-order ESO [7], [19], Generalized Proportional Integral observers (GPI) [18], [24], etc. Nevertheless these ESO designs do not take into account explicitly the effect of the noise measurement on the state observation. If some statistical information on the noise is known, a typical framework to take this information into account is the vast family of observers based on Bayesian estimation [21] like for instance Linear Kalman filter (LKF) for linear systems, Extended Kalman filter (EKF) for linearized nonlinear systems, Unscented Kalman Filter (UKF) [11] and Particule Filter (PF) [22] for nonlinear systems. Such state estimation approaches need a model to be implemented, which seems to make them totally useless if the system is unknown. The aim of this paper is to show that the most basic and aged filter of this family, eg. the discrete time-varying Linear Kalman Filter introduced in 1960, can be used to estimate the state of a given class of unknown and time-varying nonlinear SISO systems if their outputs are corrupted by a known Gaussian additive white noise. The remainder of the paper is organized as follows. Section II introduces some already published approaches that will be reused in this paper. Section III specifies the class of nonlinear systems that are considered and defines the framework of the developed approach. Section IV proves that these nonlinear systems can be described by a linear system of any order to which is added a virtual input. Section V presents the linear Kalman filter that is used to reconstruct not only the state components of the system but also the virtual input. Some simulation results are shown in section VI to exhibit the efficiency of the proposed observation method. Finally, concluding discussions are given in sections VII and VIII.

\section{ROOTS OF THE PROPOSED OBSERVATION STRATEGY}

The observation strategy presented in this paper takes its roots in the ADRC framework and also in an unknown input observation technique for known linear systems that uses a LFK [23]. For SISO systems, ADRC addresses the observation and the control of dynamical uncertain nonlinear systems of order $p$ whose state-space representation is given by [10]:

$$
\left\{\begin{aligned}
\dot{x}_{1} & =x_{2}, \\
& \vdots \\
\dot{x}_{p-1} & =x_{p}, \\
\dot{x}_{p} & =f(X, d, t)+b(X, t) u, \\
y & =x_{1},
\end{aligned}\right.
$$


where $d$ is some unknown external disturbance, $f(X, d, t) \in$ $\mathbb{R}$ and $b(X, t) \in \mathbb{R}$ are some time-varying nonlinear and possibly unknown functions with $b(X, t) \neq 0$. If $b_{0} \neq 0$ is chosen to be an estimation of $b(X, t)$, and if the total disturbance, that lumps the model uncertainties and $d$, is defined as an unknown extended state component $x_{p+1}$ :

$$
x_{p+1} \triangleq f(X, d, t)+\left(b(X, t)-b_{0}\right) u
$$

then (1) is equivalent to

$$
\left\{\begin{aligned}
\dot{x}_{1} & =x_{2}, \\
& \vdots \\
\dot{x}_{p-1} & =x_{p}, \\
\dot{x}_{p} & =x_{p+1}+b_{0} u, \\
y & =x_{1} .
\end{aligned}\right.
$$

If an extended state $X^{e}=\left[x_{1} \ldots x_{p} x_{p+1}\right]^{\top}$ is introduced, then $X^{e}$ can be classically observed with any ESO of order $p+1$ developed in the literature like Linear Extended State Observers [17], [25], [26], [16], [14] or higher-order ESO already cited that improve the estimation quality [19]. The idea of using an extended state of order $p+1$ will be keep in this paper but the proposed approach will provide an extension of the classical ADRC formulation. Using an appropriate equivalent state-space representation introduced in Section IV, the proposed approach will also consider that the extended state component is here a virtual additive unknown input. Estimating an unknown input with a LKF has been proposed in [23], but exclusively for a perfectly known linear system having a null control input. The challenge to face consists in merging these two approaches, with one related to an unknown nonlinear system and extended state and the other to a known linear system with unknown additive input and Gaussian measurement error.

\section{PROBLEM STATEMENT}

This paper addresses the state observation problem of unknown nonlinear and time-varying SISO systems that obey the three following assumptions.

Assumption 1: The systems of interest are nonlinear, unknown and time-varying, with an input-output dynamics that can be described by an Ordinary Differential Equation (ODE) of order $n>0$ defined by:

$$
y^{(n)}(t)=f\left(y(t), \ldots, y^{(n-1)}(t), u(t), U^{\prime}(t), t\right)
$$

where

- the order $n$ is unknown;

- the nonlinear and time-varying function $f$ exists but is unknown;

- the scalar output $y(t) \in \mathbb{R}$ will be sampled and measured with a zero-mean additive Gaussian noise $v\left(k T_{s}\right) \in \mathbb{R}$ with a known variance $R$.

- the scalar input $u(t) \in \mathbb{R}$ is the known piecewisecontinuous output of the controller given by a zero-order hold;
- the multidimensional piecewise-continuous disturbance inputs $U^{\prime}(t) \in \mathbb{R}^{\delta}$ are assumed unknown. The dimension $\delta$ is also unknown. These disturbances can affect the system in any way.

Assumption 2: The state vector $\mathcal{X} \in \mathbb{R}^{n}$ of the unknown system defined by (4) is written as

$$
\mathcal{X}=\left[\begin{array}{llll}
y & \dot{y} & \cdots & y^{(n-1)}
\end{array}\right]^{\top} .
$$

Assumption 3: The output $y$ and its derivatives $\dot{y}, \ldots, y^{(p-1)}$ with $p>0$ are assumed necessary to implement the control law of the unknown system. These $p$ components are gathered in the vector

$$
X=\left[\begin{array}{llll}
y & \dot{y} & \cdots & y^{(p-1)}
\end{array}\right]^{\top} \in \mathbb{R}^{p}
$$

that has to be observed, with $p$ being chosen. The strategy to choose $p$ is outside the scope of this paper that is only focused on the observation of $X$ for any chosen $p$.

With such assumptions, $p$ can be higher, equal or lower than the unknown value $n$. If $p \leq n$ then the observer will be a reduced-order or a full-order observer of the state $\mathcal{X}$. If $p>n$ then

$$
X=\left[\begin{array}{lll}
\mathcal{X}^{\top} y^{(n)} & \ldots & y^{(p-1)}
\end{array}\right]^{\top} .
$$

In this case, the $n$ state components of $\mathcal{X}$ and $p-n$ additional successive output derivatives will be observed.

\section{EQUIVALENT STATE-SPACE REPRESENTATION}

Let us introduce $p$ a priori coefficients $a_{i} \in \mathbb{R}$ and a scalar $b \neq 0$. The nonlinear $n$-order ODE (4) can be represented by an equivalent linear $p$-order ODE using the procedure explained below. From (4):

$$
y^{(n)}-f\left(y, \ldots, y^{(n-1)}, u, U^{\prime}, t\right)=0
$$

which can be written as

$$
\begin{aligned}
& y^{(n)}-f\left(y, \ldots, y^{(n-1)}, u, U^{\prime}, t\right) \\
& -y^{(p)}+\left(a_{1} y+\ldots+a_{p} y^{(p-1)}+b u\right) \\
& +y^{(p)}-\left(a_{1} y+\ldots+a_{p} y^{(p-1)}+b u\right)=0 .
\end{aligned}
$$

Let us define a virtual input $\mathscr{C} \in \mathbb{R}$ by

$$
\begin{aligned}
\mathscr{C} \triangleq & -\frac{1}{b}\left[\left(y^{(n)}-f\left(y, \ldots, y^{(n-1)}, u, U^{\prime}, t\right)\right)\right. \\
& \left.-y^{(p)}+\left(a_{1} y+\ldots+a_{p} y^{(p-1)}+b u\right)\right]
\end{aligned}
$$

such that Eq. (9) is equivalent to the following $p$-order ODE by injecting $\mathscr{C}$ in it:

$$
y^{(p)}=a_{1} y+\ldots+a_{p} y^{(p-1)}+b u+b \mathscr{C} .
$$

Thanks to (8), it is possible to simplify (10) to

$$
\mathscr{C}=-\frac{1}{b}\left[-y^{(p)}+a_{1} y+\ldots+a_{p} y^{(p-1)}+b u\right] .
$$

If $p \leq n$, the unknown temporal functions $y, \dot{y}, \ldots$, $y^{(p)}$ in (12) are mathematically defined thanks to (4). If 
$p>n$, the nonlinear function $f$ has to be $(p-n-1)$ differentiable. Then $y, \ldots, y^{(n)}$ are defined by (4) and the additional unknown temporal functions $y^{(n+1)}, \ldots, y^{(p)}$ are mathematically defined by differentiating (4) $p-n-1$ times, which lead to:

$$
\left.y^{(p)}=\frac{d^{p-n-1}}{d t^{p-n-1}} f\left(y, \ldots, y^{(n-1)}, u, U^{\prime}, t\right)\right) .
$$

As both ODEs (4) and (11) share the same first $p$ initial conditions on $y\left(t_{0}\right), \ldots, y^{(p-1)}\left(t_{0}\right),(11)$ has the same inputoutput behavior as the unknown nonlinear $n$-order ODE (4). On a structural point of view, (11) now depends on $p$ known coefficients $a_{i}$, a known coefficient $b$ and an unknown virtual input $\mathscr{C}$. With (6), the equivalent input-output dynamics (11) can be written as a state-space representation:

$$
\begin{gathered}
\dot{X}=A X+B u+B \mathscr{C} \\
y=C X, \\
A=\left[\begin{array}{cccccc}
0 & 1 & 0 & 0 & \ldots & 0 \\
0 & 0 & 1 & 0 & \ldots & 0 \\
& \ddots & & \\
0 & 0 & 0 & \ldots & 0 & 1 \\
a_{1} & a_{2} & a_{3} \ldots & a_{p-1} & a_{p}
\end{array}\right]_{p \times p} \quad B=\left[\begin{array}{c}
0 \\
0 \\
\vdots \\
0 \\
b
\end{array}\right]_{p \times 1} \\
C=\left[\begin{array}{llll}
1 & 0 & \ldots & 0
\end{array}\right]_{1 \times p} .
\end{gathered}
$$

By setting $X=\left[x_{1} \cdots x_{p}\right]^{\top}$, (14) can be put under the following form with (15):

$$
\left\{\begin{aligned}
\dot{x}_{1} & =x_{2}, \\
& \vdots \\
\dot{x}_{p-1} & =x_{p} \\
\dot{x}_{p} & =a_{1} x_{1}+\cdots+a_{p} x_{p}+b u+b \mathscr{C}, \\
y & =x_{1} .
\end{aligned}\right.
$$

If we choose to set all the $p$ coefficients $a_{i}$ to 0 and $b$ to $b_{0}$, then (16) becomes equivalent to (3) with the unknown extended state $x_{p+1}$ equal to $b \mathscr{C}$. In this paper, the choice of the parameters $a_{i}$ will not be restricted to 0 . Therefore, the standard SISO state-space formulation of ADRC can be seen as a specific case of the proposed methodology.

\section{State Observer USing Kalman Filtering}

The representation (14) reproduces the input-output behavior of the nonlinear unknown system (4). This representation of order $p$ is linear with perfectly known input, state and output matrices. As the output $y$ is measured at each sample time $t_{k}$ with an additive white gaussian zero-mean noise $v_{k} \in \mathbb{R}$ having a known variance $R$, a linear Kalman filter [12] based on matrices $A, B$ and $C$ is an appropriate way to achieve the observation of the unknown nonlinear system. Nevertheless, using such an approach implies that a Gaussian process noise can be correctly defined and that its associated covariance matrix can be mathematically derived. In most implementation of Kalman filtering, this point is tricky and lead to some heuristic choices. We show in the following that the approach previously developed in [23] for a secondorder system with $u=0$ can be adapted to solve this point in our framework.

\section{A. Virtual input modeling}

Using a Bayesian framework to establish an a posteriori unknown input estimation implies that this estimation is based on an a priory uncertain modeling of the virtual input evolution. Such modeling has to formulate that this evolution is completely unknown. In other words, whatever is $d t \neq 0$, strictly nothing can be a priori deduced on the derivative $\dot{\mathscr{C}}(t+d t)$ from the knowledge of $\dot{\mathscr{C}}(t)$. Such property can be easily hold by a modeled stochastic signal $\dot{\mathscr{C}}_{m}(t)$ with an infinite bandwidth and an auto-correlation function being a Dirac delta impulse. This modeled derivative of the input signal is defined by:

$$
\dot{\mathscr{C}}_{m}(t)=\zeta(t)
$$

with $\zeta(t)$ a zero-mean infinite-variance white Gaussian stochastic process characterized by its power spectral density (PSD) $W$ given by the auto-correlation function $\phi$ of $\zeta(t)$ :

$$
\phi_{\zeta, \zeta}(\tau)=W \delta(\tau) \quad \forall \tau \in \mathbb{R}
$$

in which $\delta(\tau)$ is a Dirac delta impulse. Whatever is the dimension $p$ of $X$, once matrices $A, B$ and $C$ are set arbitrarily, $W \in \mathbb{R}$ is the only a priori parameter to adjust that will influence the dynamics of the observer (ie the observer gain) and its level of noise.

\section{B. Discrete Kalman filter synthesis}

An extended stochastic state $X^{e}=\left[X^{\top} \mathscr{C}_{m}\right]^{\top} \in \mathbb{R}^{p+1}$ is used to introduce the modeled stochastic input $\mathscr{C}_{m}$ into the state to estimate. The associated stochastic state-space representation is obtained using (17) and (14) with $\mathscr{C}$ substituted by $\mathscr{C}_{m}$ :

$$
\begin{aligned}
\dot{X}^{e} & =\mathcal{A} X^{e}+\mathcal{B} u+\mathcal{M} \zeta, \\
y & =\mathcal{C} X^{e},
\end{aligned}
$$

$$
\mathcal{A}=\left[\begin{array}{cc}
A & B \\
0_{1 \times p} & 0
\end{array}\right] \quad \mathcal{B}=\left[\begin{array}{c}
B \\
0
\end{array}\right] \mathcal{M}=\left[\begin{array}{c}
0_{p \times 1} \\
1
\end{array}\right] \quad \mathcal{C}=\left[\begin{array}{ll}
C & 0
\end{array}\right] \text {. }
$$

A discretization using a zero-order hold (zoh) gives the evolution of $X_{k}^{e}=\left[X_{k} \mathscr{C}_{k}\right]^{\top}$, with $\mathscr{C}_{k}$, the discretization of $\mathscr{C}_{m}$ by the sampling period $T_{s}$ :

$$
\begin{gathered}
X_{k+1}^{e}=\mathcal{F} X_{k}^{e}+\mathcal{G} u_{k}+Z_{k}, \\
y_{k}=\mathcal{C} X_{k}^{e}, \\
\mathcal{F}=e^{\mathcal{A} T_{s}} \quad \mathcal{G}=\int_{0}^{T_{s}} e^{\mathcal{A} t} d t \mathcal{B} .
\end{gathered}
$$

The process noise $Z_{k} \in \mathbb{R}^{p+1}$ is a zero-mean band-limited Gaussian white noise that characterizes the uncertainty on the evolution of $X_{k}^{e}$. This uncertainty is due to the a priori stochastic modeling $\dot{\mathscr{C}}_{m}(t)$ whose infinite bandwidth is now restricted by the sampling period $T_{s}$ used in the zoh. Its $(p+1) \times(p+1)$ covariance matrix $Q$ is:

$$
\begin{aligned}
Q & =E\left[Z_{k} Z_{k}^{\top}\right]=\int_{0}^{T_{s}} e^{\mathcal{A} t} \mathcal{M} W \mathcal{M}^{\top} e^{\mathcal{A}^{\top} t} d t \\
& =W \int_{0}^{T_{s}} e^{\mathcal{A} t} \mathcal{M} \mathcal{M}^{\top} e^{\mathcal{A}^{\top} t} d t=W \eta\left(T_{s}\right) .
\end{aligned}
$$


Thus, for a given $T_{s}, W$ simply induces a proportional factor in $Q$. The matrix structure of $Q$ is given by $\eta\left(T_{s}\right)$. This means that the uncertainty on the extended state evolution will raise if the parameter $W$ increases. The covariance $Q$ can be computed using a numerical or a symbolic solver. The measurement $y_{k}^{m}$ of $y_{k}$ is assumed to be given by

$$
y_{k}^{m}=y_{k}+v_{k}
$$

with $v_{k}$ a discrete-time band-limited gaussian white noise with zero-mean and variance $R=E\left[v_{k}^{2}\right]$. Using an a priori initial extended state estimation $\hat{X}_{0}^{e}$ and error estimation covariance matrix $P_{0}$ (that only influences the transient response of the observer from its initial state), the classical predict and update phases of the discrete time-varying Kalman filter are given by:

$$
\begin{aligned}
& \hat{X}_{k \mid k-1}^{e}=\mathcal{F} \hat{X}_{k-1}^{e}+\mathcal{G} u_{k-1}, \\
& P_{k \mid k-1}=\mathcal{F} P_{k-1} \mathcal{F}^{\top}+Q, \\
& K_{k}=P_{k \mid k-1} \mathcal{C}^{\top}\left(\mathcal{C} P_{k \mid k-1} \mathcal{C}^{\top}+R\right)^{-1}, \\
& \hat{X}_{k}^{e}=\hat{X}_{k \mid k-1}^{e}+K_{k}\left(y_{k}^{m}-\mathcal{C} \hat{X}_{k \mid k-1}^{e}\right), \\
& P_{k}=\left(I_{(p+1) \times(p+1)}-K_{k} \mathcal{C}\right) P_{k \mid k-1},
\end{aligned}
$$

provided that the pair $(\mathcal{F}, \mathcal{C})$ is observable. The first $p$ components of $\hat{X}_{k}^{e}$ give the estimation $\hat{X}_{k}$ of $X(t)$ at time $t=k T_{s}$ and its last component gives the estimation $\hat{\mathscr{C}}_{k}$ of $\mathscr{C}(t)$. Note that the scalar parameter $W$ in (18) adjusts a trade-off between the response time of the observer and its variance for each extended state component [23]. If $W$ increases, the response time of the observer decreases but its level of noise increases.

\section{ILLUSTRATIVE EXAMPLE}

In order to illustrate the efficiency of the proposed approach, the latter is applied to an unknown complex nonlinear and time-varying system, with unknown additive and multiplicative disturbances.

\section{Description of the system}

The behavior of the unknown system is described by the following equation:

$$
\begin{aligned}
y^{(4)} & =-0.1 y^{(3)}-5 \ddot{y}-2.5 \operatorname{sgn}(y) \operatorname{arctanh}\left(\dot{y}^{2}\right) y+d_{1} \\
& +0.3 \arccos y-0.01 \arctan \left(e^{y}\right) \sin (0.1 t)+d_{2} u .
\end{aligned}
$$

The unknown time-varying disturbances $U^{\prime}(t)$ are:

$$
\left[\begin{array}{ll}
d_{1} & d_{2}
\end{array}\right]^{\top}=\left[\begin{array}{ll}
0.1 \sin t & e^{-0.005 t}
\end{array}\right]^{\top} \text {. }
$$

It has to be noted that (33) and (34) are only given for information and are unknown to the observation process. Also note that the disturbance $d_{2}$ changes the applied control input in a varying multiplicative way that is unknown to the observer. The disturbed input $d_{2} u$ as stated in (33) is plotted in red in Fig. (1b). In addition to the nonlinear functions applied on $y$ and its derivatives, the output $y$ is also multiplied by a function of $\dot{y}$ and a sin function brings a time-varying behavior in the ODE.

\section{Choice of the LTI model}

Let us arbitrarily choose $p=2$ to observe the state components $X=\left[\begin{array}{ll}x_{1} & x_{2}\end{array}\right]^{\top}=\left[\begin{array}{ll}y & \dot{y}\end{array}\right]^{\top}$. To illustrate the fact that the dynamics behavior associated to the LTI coefficients is not a critical point, a very underdamped but sufficiently fast LTI system is chosen arbitrarily to observe the unknown nonlinear system, which leads to the following matrices $A$ and $B$ :

$$
A=\left[\begin{array}{cc}
0 & 1 \\
a_{1} & a_{2}
\end{array}\right], \quad B=\left[\begin{array}{l}
0 \\
b
\end{array}\right]
$$

where $a_{1}=-1.40, a_{2}=-0.05, b=450$. The control input $u(t)$ is a step input of magnitude 0.005 at time $t=20 \mathrm{~s}$.

The equivalent representation of the nonlinear system (4) is illustrated in Fig. (1a). It shows the output of the different representations of the system for the input $u(t)$ and for null initial conditions. First, the output of the underdamped LTI system (35) is displayed in magenta. It is null for the first 20 seconds. Second, the output of the same LTI system with the virtual input $\mathscr{C}$, denoted LTI $+\mathscr{C}$, is pictured in cyan. It is complex and not null for the first 20 seconds. Finally, the output of the nonlinear system in (33) is represented in black. From figure (1a), it is clear that the dynamics of the nonlinear system (33) is the same as the dynamics of the $\mathrm{LTI}+\mathscr{C}$ system (14).

\section{Configuration of the Kalman filter}

The initial a priori value of the extended state estimation has been chosen arbitrarily to $\hat{X}_{0}^{e}=\left[\begin{array}{lll}1.5 & 0 & 0\end{array}\right]$ (with wrong initial value for $x_{1}(0)$ and $\mathscr{C}(0)$ ), the power spectral density to $W=7.10^{-8}$ and the error estimation covariance matrix to $P_{0}=\operatorname{diag}\left[10^{-5} ; 10^{-5} ; 5 \cdot 10^{-10}\right]$. A small matrix $P_{0}$ will induce a large confidence in $\hat{X}_{0}^{e}$ and thus a slow transient response of the observer to correct the errors present in its initial condition $\hat{X}_{0}^{e}$. Furthermore, the output measurement of the nonlinear system (33) is affected by a noise $v_{k}$, which is a band-limited Gaussian white noise, with zero-mean and variance $R=10^{-5}$ (see Fig. (1b)). The estimation results of the Kalman filter of the extended state $X^{e}=\left[\begin{array}{lll}y & \dot{y} & \mathscr{C}\end{array}\right]^{\top}$ with a sampling time $T_{s}=0.01 \mathrm{~s}$ are illustrated in Figures (1c), (1d) and (1e). In these three figures, the real component of the extended state is displayed in red while its estimation is plotted in blue. The real value of $\mathscr{C}$ is computed using (12). In the first seconds, the wrong initial estimations for $x_{1}(0)$ and $\mathscr{C}(0)$ are rapidly corrected by the observer. Then, one can see that, despite the noisy measurement of the complex nonlinear system's output, the linear Kalman filter, built on a simple 2nd-order LTI system having a sufficient bandwidth, is able to track the first two states components of the nonlinear system (33) and $\mathscr{C}$ with a bounded error given in Fig. (1f).

\section{DISCUSSION AND PERSPECTIVE}

If no information is available on the system, the coefficients $a_{i}$ and $b \neq 0$ can only be chosen randomly (whereas in common ADRC, these coefficients are necessary null). 


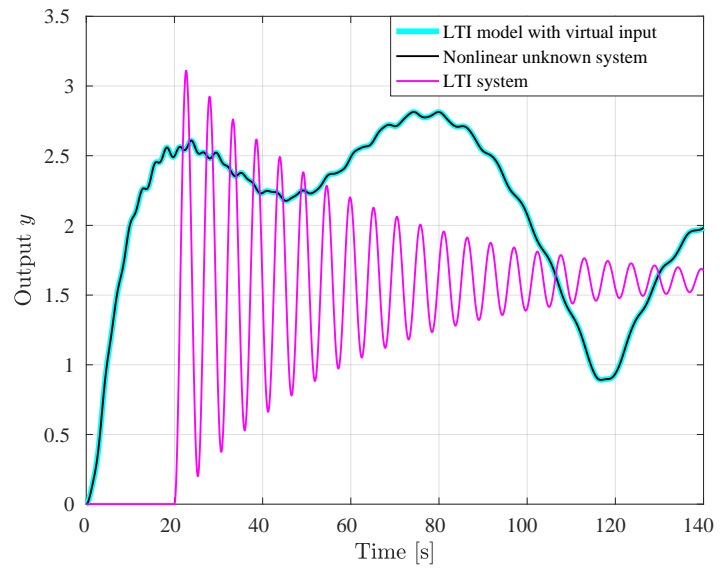

(a) Comparison of the behavior of the output $y$

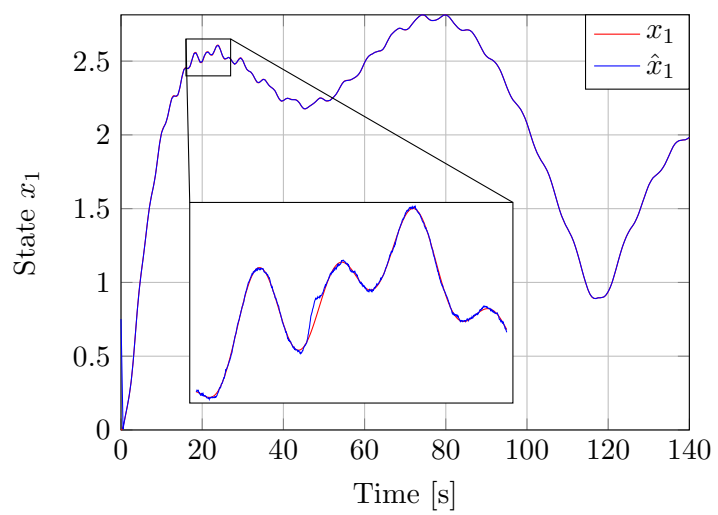

(c) Evolution of the state $x_{1}$ and its estimation

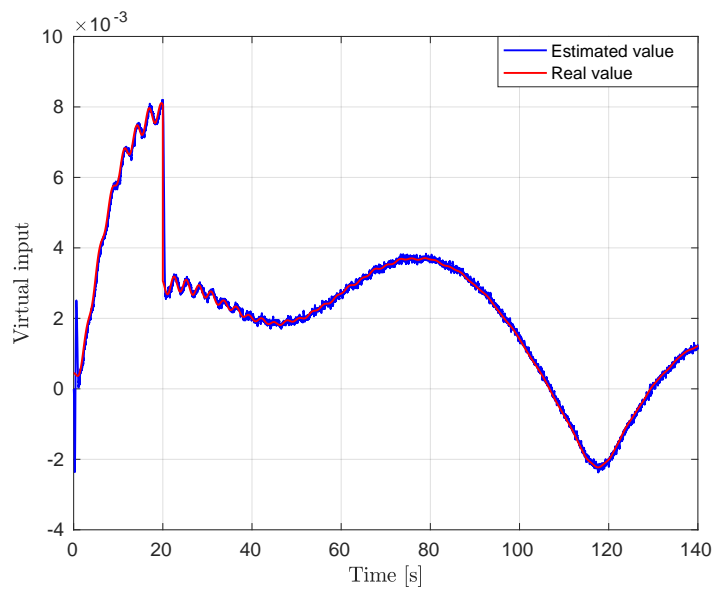

(e) Evolution of the virtual input and its estimation

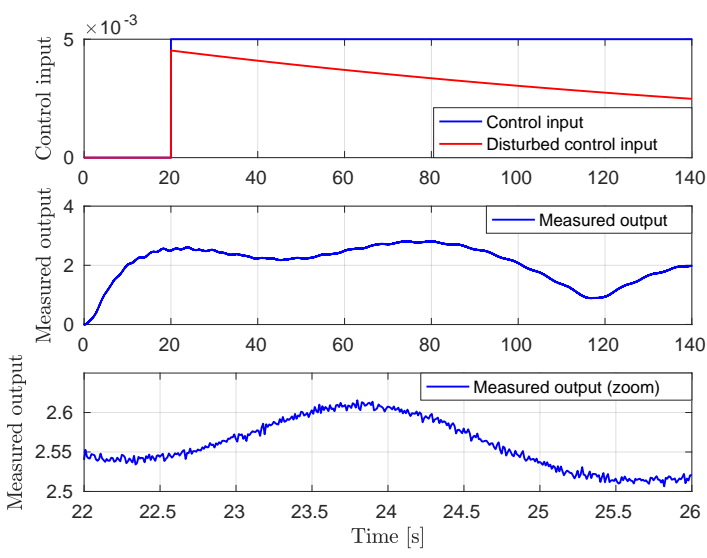

(b) Evolution of the control input, evolution of the measured output and its zoom

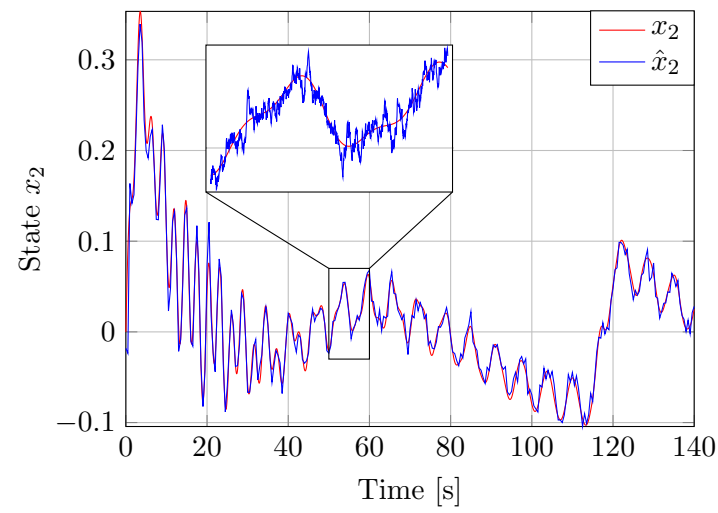

(d) Evolution of the state $x_{2}$ and its estimation
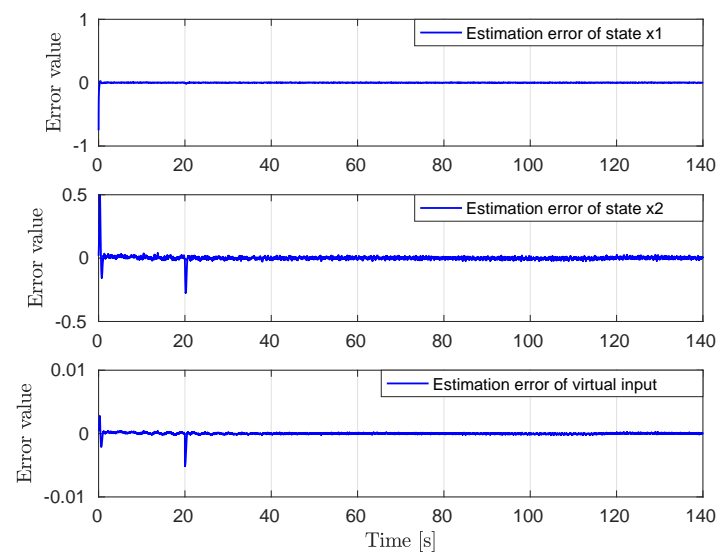

(f) Evolution of the estimation errors

Fig. 1: Simulation results 
If an approximate LTI modeling compatible with matrices (15) is available, it can be used to set prior values. Whatever is the strategy chosen, the choice made will influence the observer efficiency. Model-free optimization techniques may be used to enhance the observer efficiency with dynamical coefficients $a_{i}$ and this point has to be studied in the future. The choice of $T_{s}$ has also to be coherent with the bandwidth of the system. Then, the parameter $W$ has to be tuned to adjust the level of noise in $\hat{\mathscr{C}}_{k}$. As shown in [23], smaller is $W$ and smaller is the observer dynamics, but also smaller is the noise in the observation. Furthermore, if $W$ is chosen too small, a delay appears in the observation of the high part of the spectrum of $\mathscr{C}$ and $X$. This may lead to an unstable closed loop when the control is added. To enhance the efficiency of LKF, it should be interesting, like for ESO, to develop higher-order extended state estimation by adding the successive derivatives of $\mathscr{C}$ into the extended state $X^{e}$. This should give a better estimation of the dynamics of $\mathscr{C}$ and thus a better estimation of $X$. This issue is also a perspective for this work.

\section{CONCLUSION}

Providing simple, efficient and generic designs for the observation and control of complex unknown nonlinear systems is a challenging issue in control theory. The proposed methodology proposes an alternative to Extended States Observers used in ADRC when the variance of the noise measurement is exploited to perform the observation of a specific class of nonlinear and time-varying SISO systems with unknown order, structure and disturbances. It generalizes the use of linear Kalman filtering for this class of unknown systems. It also provides a straightforward mathematical background to define the process noise and its covariance matrix while these points are generally tricky to define in conventional implementations of Kalman filters. This illustrates that the potential of linear Kalman filtering for nonlinear systems is much larger than commonly admitted. It also illustrates that a model-based linear observer can be used to observe totally unknown nonlinear systems for which no model is available. The arbitrary LTI model used in the LKF can be very different than the system physical behavior. The important parameter to tune in the observer is a scalar power spectral density that is related to the effective observer bandwidth, delay and noise.

\section{REFERENCES}

[1] Yali Dong, Hui Wang, and Yangang Wang. Design of Observers for Nonlinear Systems with $\mathrm{H}_{\infty}$ Performance Analysis. Mathematical Methods in the Applied Sciences, 37(5):718-725, 2013.

[2] Guang Feng, Yan-Fei Liu, and Lipei Huang. A New Robust Algorithm to Improve the Dynamic Performance on the Speed Control of Induction Motor Drive. IEEE Transactions on Power Electronics, 19(6):1614-1627, 2004.

[3] Zhiqiang Gao. Scaling and Bandwidth-Parameterization Based Controller Tuning. In Proceedings of the American Control Conference, Denver, CO, USA. IEEE, 2003.

[4] Zhiqiang Gao. Active Disturbance Rejection Control: A Paradigm Shift in Feedback Control System Design. In Proceedings of the American Control Conference, Minneapolis, MN, USA. IEEE, 2006.
[5] Zhiqiang Gao, Yi Huang, and Jingqing Han. An Alternative Paradigm for Control System Design. In Proceedings of the 40th Conference on Decision and Control, Orlando, FL, USA. IEEE, 2001.

[6] Jean-Paul Gauthier, Hassan Hammouri, and Sami Othman. A Simple Observer for Nonlinear Systems - Applications to Bioreactors. IEEE Transactions on Automatic Control, 37(6):875-880, 1992.

[7] Ashwini A. Godbole, Jaywant P. Kolhe, and Sanjay E. Talole. Performance Analysis of Generalized Extended State Observer in Tackling Sinusoidal Disturbances. IEEE Transactions on Control Systems Technology, 21(6):2212-2223, 2013.

[8] Bao-Zhu Guo and Zhi-Liang Zhao. Active Disturbance Rejection Control for Nonlinear Systems: An Introduction. Wiley, 2016.

[9] Jingqing Q. Han. The Extended State Observer for a Class of Uncertain Systems. Control and Decision, 10(1):85-88, 1995.

[10] Yi Huang and Wenchao Xue. Active Disturbance Rejection Control: Methodology and Theoretical Analysis. ISA Transactions, 53(4):963976, 2014.

[11] Simon J. Julier and Jeffrey K. Uhlmann. A New Extension of the Kalman Filter to Nonlinear Systems. In Proceedings of AeroSense: 11th International Symposium on Aerospace / Defense Sensing, Simulations and Controls, Orlando, FL, USA. SPIE, 1997.

[12] Rudolf E. Kalman. A New Approach to Linear Filtering and Prediction Problems. Journal of Basic Engineering, 82(1):35-45, 1960.

[13] Hassan K. Khalil and Laurent Praly. High-Gain Observers in Nonlinear Feedback Control. International Journal of Robust and Nonlinear Control, 24(6):993-1015, 2013.

[14] Kyung-Soo Kim, Keun-Ho Rew, and Soohyun Kim. Disturbance Observer for Estimating Higher Order Disturbances in Time Series Expansion. IEEE Transactions on Automatic Control, 55(8):19051911, 2010.

[15] Arthur J. Krener and Alberto Isidori. Linearization by Output Injection and Nonlinear Observers. Systems \& Control Letters, 3(1):47-52, 1983.

[16] Shihua Li, Jun Yang, Wen-Hua Chen, and Xisong Chen. Generalized Extended State Observer Based Control for Systems with Mismatched Uncertainties. IEEE Transactions on Industrial Electronics, 59(12):4792-4802, 2012.

[17] Shihua Li, Jun Yang, Wen-Hua Chen, and Xisong Chen. Disturbance Observer-Based Control - Methods and Applications. CRC Press, 2014.

[18] Alberto Luviano-Juárez, John Cortés-Romero, and Hebertt írez. Synchronization of Chaotic Oscillators by Means of Generalized Proportional Integral Observers. International Journal of Bifurcation and Chaos, 20(5):1509-1517, 2010.

[19] Rafal Madoński and Przemyslaw Herman. Survey on Methods of Increasing the Efficiency of Extended State Disturbance Observers. ISA Transactions, 56:18-27, 2015.

[20] Lorenzo Marconi, Laurent Praly, and Alberto Isidori. Output Stabilization via Nonlinear Luenberger Observers. SIAM Journal on Control and Optimization, 45(6):2277-2298, 2007.

[21] Peter S. Maybeck. Stochastic models, estimation, and control. ACADEMIC Press, 1982.

[22] Pierre Del Moral. Nonlinear Filtering: Interacting Particle Solution. Markov Processes and Related Fields, 2(4):555-580, 1996.

[23] Emmanuel Piat, Joël Abadie, and Stéphane Oster. Nanoforce Estimation based on Kalman Filtering and Applied to a Force Sensor using Diamagnetic Levitation. Sensors and Actuators, 179:223-236, 2012.

[24] Hebertt Sira-Ramírez, Alberto Luviano-Juárez, Mario Ramírez-Neria, and Eric William Zurita-Bustamante. Active Disturbance Rejection Control of Dynamic Systems - A Flatness Based Approach. Butterworth-Heinemann, 2017.

[25] Sanjay E. Talole, Jayawant P. Kolhe, and Srivijay B. Phadke. Extended-State-Observer-Based Control of Flexible-Joint System with Experimental Validation. IEEE Transactions on Industrial Electronics, 57(4):1411-1419, 2010.

[26] Junxiao Wang, Shihua Li, Jun Yang, Bin Wu, and Qi Li. Extended State Observer-Based Sliding Mode Control for PWM-Based DC-DC Buck Power Converter Systems with Mismatched Disturbances. IET Control Theory \& Applications, 9(4):579-586, 2015.

[27] Yuanqing Xia, Zheng Zhu, Mengyin Fu, and Shuo Wang. Attitude Tracking of Rigid Spacecraft with Bounded Disturbances. IEEE Transactions on Industrial Electronics, 58(2):647-659, 2011.

[28] Michael Zeitz. The Extended Luenberger Observer for Nonlinear Systems. Systems \& Control Letters, 9(2):149-156, 1987. 